\title{
An animal restraint for the study of high-resistance operants in the rat
}

\author{
WILLIAM M. BENEKE and BENJAMIN F. TIMSON \\ Lincoln University of Missouri, Jefferson City, Missouri
}

\begin{abstract}
An animal restraint system and response operandum suitable for the study of operant behavior in the rat is described. This system combined with an inexpensive Commodore microcomputer and Psychronix interface is well-suited for the study of response parameters. Rats voluntarily entered the restraint, showed no observable signs of excess stress, and were easily extricated at the end of the experimental session. A lever-pressing operant with an adjustable force requirement was readily shaped using automated procedures. Although this system was designed for the study of high-resistance operants, it has potential advantages for other operant applications, including studies of visual discrimination and experiments requiring restraint due to physiological recording.
\end{abstract}

With few exceptions, an unrestrained animal model has been used in studies of operant behavior with the rat. The vast majority of these studies have been conducted in experimental chambers that are similar in concept if not in details of design to the original operant chambers utilized by B. F. Skinner (e.g., Skinner, 1938). Although the original apparatus design may have been primarily influenced by the desire to save labor (Skinner, 1979), this unrestrained animal approach has advantages that have left it virtually unchanged for over 50 years. In addition to reduced experimenter labor, these include the selection of the leverpress operant that has sufficient spontaneous occurrence to be readily conditioned, yet with not so high a frequency as to obscure the effects of reinforcement (Skinner, 1938). In the confines of the operant chamber, competing behaviors are limited, and stimuli are readily controllable.

The use of a restrained animal model has at least three potential advantages over the standard operant chamber for general studies of operant behavior: enhancement of stimulus control, elimination of competing behaviors, and restriction of response topography. The major advantage of the restraint in our laboratory lies in the ability of the experimenter to study operants having a specific topography. In our research, we have been concerned with the development of an animal model for studying the effects of high-resistance exercise. In addition to being interested in the variables controlling rate, duration, magnitude, and intensity of high-resistance operants, one of us (B.F.T.) is an exercise physiologist interested in cellular mecha-

Benjamin F. Timson is now in the Department of Biomedical Sciences at Southwest Missouri State University. Apparatus development and research were supported by NIH/DRR(MBRS) Grant RR-08202-05 awarded to Lincoln University. Reprint requests should be addressed to William M. Beneke, Department of Social and Behavioral Sciences, Lincoln University, Jefferson City, MO 65101 nisms of muscle hypertrophy produced by high-resistance exercise.

Exercise-physiology research in this area has suffered for lack of an animal model needed to do controlled experiments. Research with human subjects has been compromised by lack of control over motivational variables and potential confounding by variables affecting muscle growth and development that occur outside of the experimental setting. Lack of an adequate animal model has also placed ethical limits on variables that can be studied. The use of steroids in conjunction with various exercise prescriptions cannot ethically be studied, because of the health risk associated with steroid use. Analysis of the effects of the variables that can ethically be manipulated in man are limited to the analysis of body fluids and biopsy tissue; entire muscles cannot be removed for cellular analysis.

Animal models of high-resistance exercise have been developed using the cat (Gonyea \& Ericson, 1976) and the rat (Ho et al., 1980; Klitgaard, 1988; Wong \& Booth, 1988). These models have had limited success in producing muscle enlargement of the magnitude normally seen in human power lifters and body builders. An important factor in the study of muscle enlargement is the use of an intraanimal control to eliminate the genetic effect on muscle fiber number as a confounding variable. This requires that the high-resistance operant be limited to only one limb, so that the contralateral limb can be used as a control. The models of Ho et al. (1980) and Klitgaard (1988) require the rat to exercise with both limbs and are therefore limited in their ability to control genetic variables. A major limitation of the cat model (Gonyea \& Ericson, 1976) is the cost of animal care and housing.

We have developed a procedure and apparatus that eliminate some of these problems. The restraint combines the design concepts of an early device developed by Hall, Clayton, and Mark (1966) and a commercially available Acrylic Restrainer marketed by Harvard Bioscience (cat 
52-0916). Development of our restraint incorporating a response operandum and computer control system has yielded a rat model for the study of high-resistance exercise as an operant and its effects on muscle tissue. In the present paper, we describe the restraint, operandum, and microcomputer control system designed for the study of high-resistance operants.

\section{APPARATUS}

The apparatus consists of an animal restraint system that can be housed inside an animal chamber designed for a standard operant chamber. A BRS/LVE liquid solenoid valve mounted on the outside of the enclosure can be readily programmed to deliver $50 \mu$ of $10 \%$ sucrose solution, which serves as an adequate reinforcer. To eliminate the delay of reinforcement associated with transit time of the liquid from the valve to the dispenser, we attached a hypodermic needle to the "animal" end of the tube, and filled the tube with liquid at the beginning of each day. Opening the valve reliably results in a drop of liquid being dispensed from the needle. The expedience of inserting the needle into a standard drinking tube with a bearing in the end allows the animal to receive the reinforcer by licking the end of the tube.

\section{Restraint System}

The restraint, shown in Figure 1, consists of three Plexiglas parts mounted on an Alice Chatham King sling suit frame. The animal support is a flat sheet of 4-mmthick Plexiglas, $625 \times 150 \mathrm{~mm}$. A $90^{\circ}$ bend was made in the Plexiglas $380 \mathrm{~mm}$ from the end, to provide a vertical surface $150 \mathrm{~mm}$ wide and $245 \mathrm{~mm}$ high, which was suitable for mounting a houselight and cuelight. A $20 \times 52 \mathrm{~mm}$ rectangular hole was cut $48 \mathrm{~mm}$ from one side and $100 \mathrm{~mm}$ from the vertical surface to permit one foreleg access to the response operandum. A second rectangular hole, $55 \times 104 \mathrm{~mm}$, was cut $67 \mathrm{~mm}$ from the rear edge. This opening was covered with hardware cloth to permit passage of feces and urine, a feature necessary to conduct 60-min experimental sessions. The animal restraint was fashioned from 3-in. (73-mm inside diameter) Plexi-

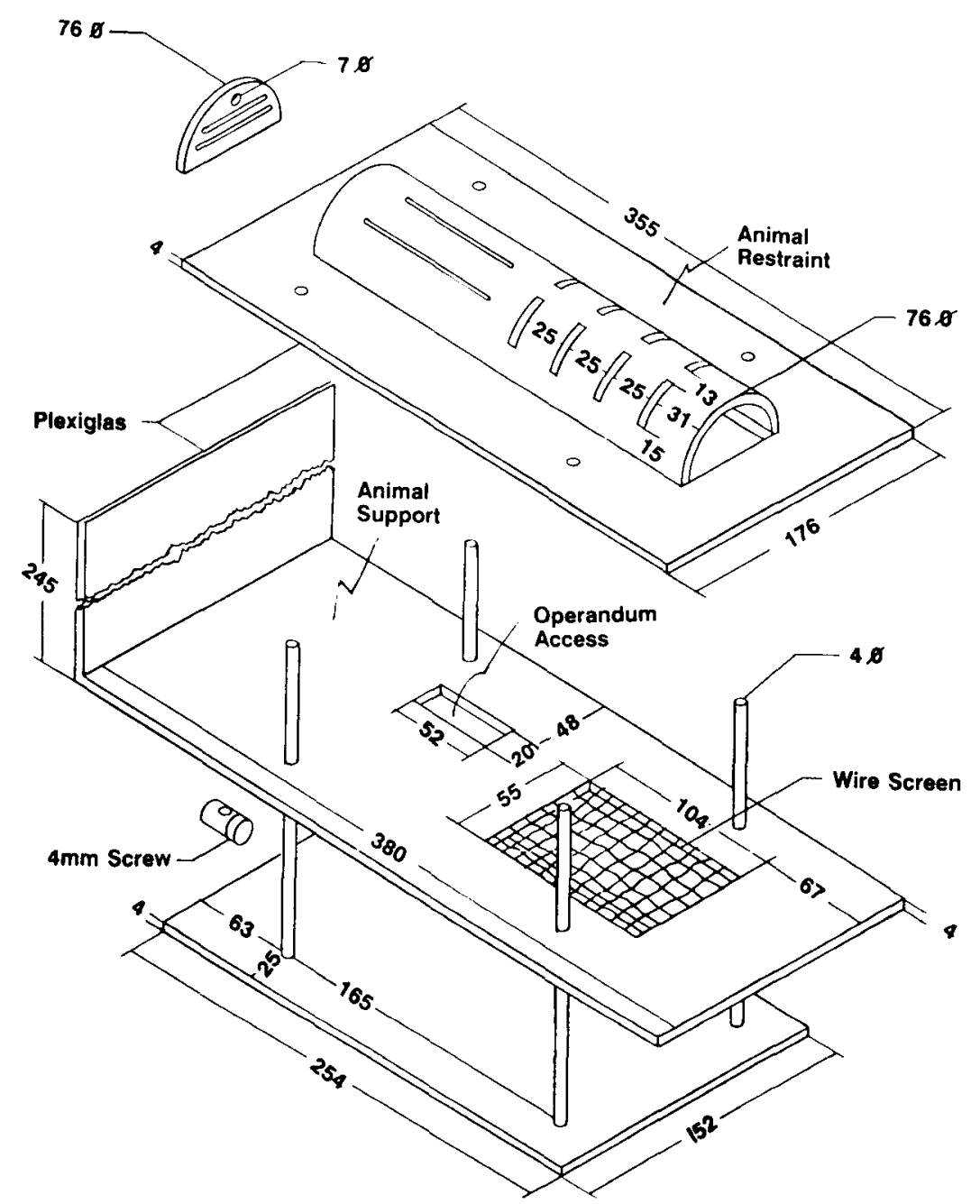

Figure 1. Restraint system for the study of operant behavior in the rat. All dimensions are in millimeters. 
glas tubing by making the longitudinal slice in the tube shown in Figure 1.

Longitudinal slots were cut in the tube to provide ventilation and cross-sectional slots to receive the tail stop (not shown). A Plexiglas piece with horizontal ventilation slots and a $7-\mathrm{mm}$ hole, drilled at a $30^{\circ}$ angle to accept a standard stainless steel drinking tube (not shown), was glued to the front of the restraining tube, closing it on that end. The tube was then glued to a $176 \mathrm{~mm}$ wide $\times 355 \mathrm{~mm}$ long rectangular Plexiglas frame. ${ }^{1}$ Drilling four holes in the animal restraint and animal support allowed both to be mounted on a rodent sling frame (from Alice King Chatham Medical Arts) and held together with the screw clamps that are supplied with the frame. Removing the top clamps allowed separation of the animal restraint and animal support for easy removal of the rat and for periodic cleaning of the apparatus. The tail stop consisted of a $67 \times 76 \mathrm{~mm}$ Plexiglas rectangle with a $16 \times 57 \mathrm{~mm}$ slot to accommodate the rat's tail. It was inserted into one of the restraint slots to prevent the rat from backing out of the restraint system during the experimental session.

\section{Operandum}

The response operandum, shown in Figure 2, consisted of a $70 \times 40 \mathrm{~mm}$ rectangular Plexiglas "paddle" glued to one end of a $3.2-\mathrm{mm}$-diam steel rod $89 \mathrm{~mm}$ from a pivot (to center of paddle). A $100-\mathrm{k} \Omega$ linear potentiometer, mounted in a Plexiglas frame, served as the pivot. The steel rod extended for $108 \mathrm{~mm}$ on the other side of the pivot, thus allowing the force necessary to depress the paddle to be varied by attachment of lead weights of varying mass at different distances from the pivot.

Depressing the paddle with sufficient force rotated the potentiometer, altering its resistance. The response operandum was attached to the base of the rodent sling frame. It was positioned so that the paddle was directly below the access hole in the animal support and the lead weight was accessible in front of the vertical portion of the animal support.

\section{Microcomputer Control}

The apparatus is controlled by a Commodore 64 microcomputer and Psychronix Interface (Crossman, 1984). Resistance from the operandum's potentiometer was applied across Pins 8 and 9 of Joystick Port 1 on the Commodore 64 microcomputer. A digital value corresponding to the paddle position (resistance of the potentiometer) was obtained using a machine language subroutine (see lines 5,000-7,040 in Appendix A) that was originally described in the Commodore 64 Programmer's Reference Guide (Commodore, 1982). This digitized index of paddle position can be utilized to measure excursion (change in paddle position over time) of the paddle-press operant, and it enables differential reinforcement of specific excursions. Control of houselights, cuelights, and liquid reinforcement is accomplished with the Psychronix interface. Data are written directly to disk. This permits alternative off-line analyses of a session-for example, several interresponse time distributions with differing bin widths are easily obtainable from a single session's data. Writing data directly to disk also permits using this system within the $5 \mathrm{~K}$ memory limitations of a less expensive VIC 20 microcomputer should one be available. All programming, other than the machine language subroutine to read paddle values, was done in BASIC. Appendix A shows a sample program listing for an alternative fixed-time 20" fixed ratio 1 (alt FT20" FR1) reinforcement schedule that we used to establish the paddle-press operant. Documentation in the listing should be removed to speed program execution. Lines 10 through 120 initialize the interface and create the disk file for the session; the program assumes that each animal has its own data disk. As events occur, a string variable consisting of a character identifying the event and the clock reading (1/60-sec resolution) is created and written to disk (cf.

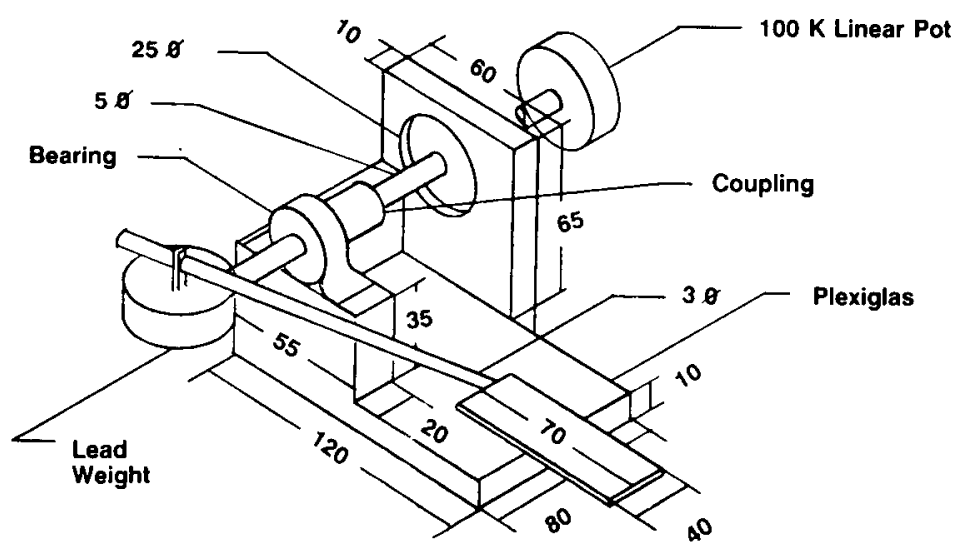

Figure 2. Response operandum used in conjunction with animal restraint. Lead weight is not to scale. Dimensions are in millimeters. Consistent with drafting conventions, zeros to the right of the decimal are indicated as slashed. 
lines 160-170). The main portion of the program goes through a loop (lines 150-280), checking for a response (lines 210-220) and $20 \mathrm{sec}$ of elapsed time (lines 230-240). The session ends after 50 reinforcers are delivered (line 270), exiting the loop for an end of session process.

\section{DISCUSSION}

We have used this apparatus to train 32 rats, collecting data over more than 50 daily 20 -min sessions for each animal, and we have experienced no serious problems related to the equipment. Nor did we observe any physical ill effects on any of the animals. There was no evidence of bleeding, nor of torn claws; nearly all the feces and urine produced in a session did pass through the hardware cloth, and any that remained was easily wiped up after removal of the rat. Adaptation to the animal restraint system and magazine training was readily accomplished in one session in which 50 reinforcers were delivered on a fixed-time (FT20") schedule. During this session, responses had no effect. Following this initial session, the paddle-press operant was established, using an alt FT20" FR1 schedule to deliver 50 reinforcers per day. Force required for the operant was set to a $.98 \mathrm{~N}$, a minimal value for the apparatus; this force was applied through a distance required to produce complete extension of the elbow joint in individual animals by software control (the typical distance was approximately $35 \mathrm{~mm}$ ). The alt FT20" FR1 schedule was in effect until $75 \%$ of the reinforcers obtained in a session were delivered as a result of the FR1 component of the schedule. The paddle-press operant was established in a mean of 6.9 sessions, with no animal requiring more than 16 sessions. By the end of the third session, all animals readily entered the restraining system. The handler simply positioned the rat facing the open end of the restraint, allowing the rat to crawl into the restraint. All the handler had to do was center the rat's tail and insert the tail stock. Animals would not, however, voluntarily back out of the restraint at the end of their experimental sessions. Removal of the top restraining part left the rat on the horizontal animal support, where a handler could easily pick him up. This made extrication of the animals a simple task. Taken together, these observations of the voluntary entry and the need to remove the top part of the restraint to extricate the animal suggest that the restraint was not aversive and likely not stressful either; this is probably the result of the use of positive reinforcement. ${ }^{2}$ Studies using aversive control with this apparatus might be expected to experience minor problems in getting subjects to enter the apparatus.

Although such a restraint system was required for our research, the advantages of the restraint should make it a useful apparatus for other studies. It would appear that mounting visual displays in the solid end of the restraint would make this restraint a valuable tool for the study of visual discrimination in the rat. The animal's eyes would always be oriented toward the display, maximiz- ing the detection of visual stimuli. This restraint may also prove beneficial in situations in which general limitations on movement are necessary, such as physiological studies in which excessive activity could interfere with physiological recording (Hall et al., 1966).

Several restraining devices for rats have been described in the psychological literature (see Hall et al., 1966, for references). In spite of their potential advantages, these devices appear to be have been used limitedly, in studies of aversive control (e.g., Azrin, Hopwood, \& Powell, 1967; Dallemagne \& Richelle, 1970) and in studies involving physiological recording (Hall et al., 1966). One can only speculate that problems of adapting animals to restraining systems, daily placement in and removal from the restraint, concerns that restraint systems would induce stress, and lack of a commercially available restraint system have been major factors in selection of the operant chamber over a restraining device. Our restraint appears to minimize all but the last problem. Though they were not commercially available, we were able to have five restraints constructed for less than $\$ 100$ per system (excluding the sling suit frames and liquid solenoid valves, which we already possessed). Although our animals were not examined for internal symptoms of stress, careful daily observation indicated no physical injuries, good health over the duration of the study, and consistent voluntary restraint entry after about 3 days of training. These observations strongly suggest that the restraint was not aversive and probably not more stressful than standard operant chambers.

\section{REFERENCES}

Azrin, N. H., Hopwood, J., Powell, J. (1967). A rat chamber and electrode procedure for avoidance conditioning. Journal of the Experimental Analysis of Behavior, 10, 291-298.

Commodore Business Machines, Inc. (1982). Commodore 64 programmer's reference guide. Westchester, PA: Author.

Crossman, E. K. (1984). An inexpensive operant chamber interface for the VIC 20 microcomputer. Behavior Research Methods, Instruments, \& Computers, 16, 338-340.

Dallemagne, G., \& Richelle, M. (1970). Titration schedule with rats in a restraining device. Journal of the Experimental Analysis of Behavior, 13, 339-348.

GONYEA, W. J., \& ERICsON, G. C. (1976). Experimental model for study of exercise-induced skeletal muscle hypertrophy. Journal of Applied Physiology, 40, 630-633.

Hall, R. D., Clayton, R. J., \&ark, R. G. (1966). A device for the partial restraint of rats in operant conditioning studies. Journal of the Experimental Analysis of Behavior, 9, 143-145.

Ho, K. W., Roy, R. R., TWeedle, C. D., Heusner, W. W., Van Huse, W. D., \& CARROW, R. E. (1980). Skeletal muscle fiber splitting with weight lifting exercise in rats. American Journal of Anatomy, 157, 433-440.

KLITGAARD, H. (1988). A model for quantitative strength training of hindlimb muscles of the rat. Journal of Applied Physiology, 64, 1740-1745.

SKINNER, B. F. (1938). The behavior of organisms. New York: Appleton.

SKINNER, B. F. (1979). The shaping of a behaviorist. New York: Alfred A. Knopf.

WoNG, T. S., \& BoorH, F. W. (1988). Skeletal muscle enlargement with weight-lifting exercise by rats. Journal of Applied Physiology, $65,950-954$. 


\section{NOTES}

1. Since the restraint is designed to be removed from the animal support, making this frame wider than the support provides a convenient handle that facilitates removal.

2. An earlier version of this restraint system was constructed with the restraint permanently attached to the support. In a pilot study with the earlier version and a water reinforcer, removal of the rat at the end of the session became progressively more difficult, eventually requiring more time than was required for the entire experimental session. The removable restraint eliminates this problem and has the added advantage of facilitating cleaning.

Appendix A

Program Listing for alt FT20" FR1*

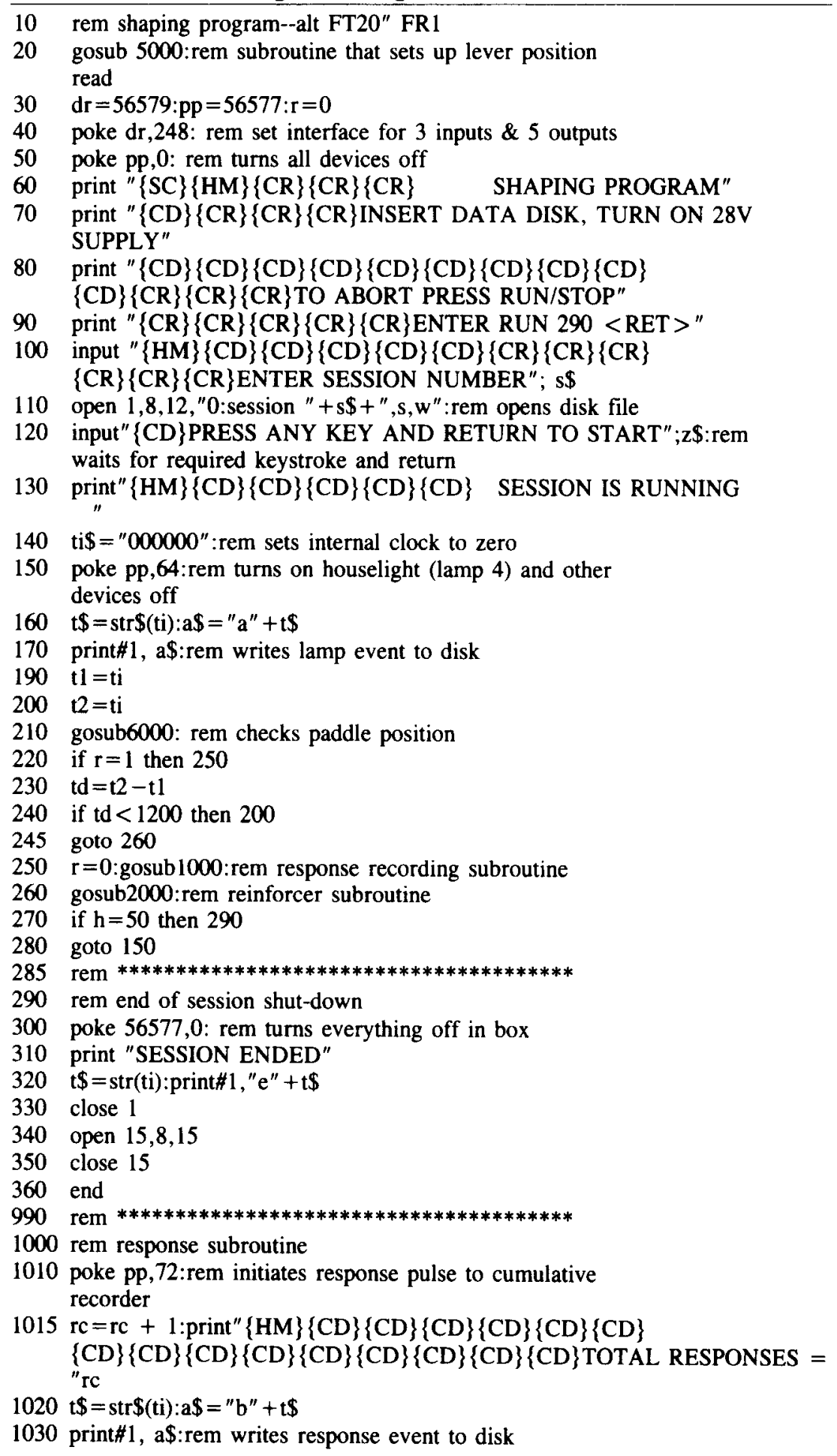


1040 for $i=1$ to 9 :next

1050 poke $\mathrm{pp}, 64$ :rem ends response pulse

1060 return

1070 rem

2000 rem reinforcer subroutine

2005 t\$ = str\$(ti):print $\# 1, " c^{\prime \prime}+t \$$ :rem writes reinforcer event to disk

2010 poke pp,208:rem turns on liquid solenoid valve for reinforcer; cue light on; leaves house light on

2020 for $\mathrm{i}=1$ to 40 :next: rem allows pulse duration to deliver $.5 \mathrm{ml}$ reinforcer

2030 poke pp,80: rem turns off reinforcer valve, cue light continues

$2040 \mathrm{~h}=\mathrm{h}+1$ :rem increments reinforcer counter

2050 print" $\{\mathrm{HM}\}\{\mathrm{CD}\}\{\mathrm{CD}\}\{\mathrm{CD}\}\{\mathrm{CD}\}\{\mathrm{CD}\}\{\mathrm{CD}\}\{\mathrm{CD}\}\{\mathrm{CD}\}\{\mathrm{CD}\}$ $\{\mathrm{CD}\}\{\mathrm{CR}\}\{\mathrm{CR}\}\{\mathrm{CR}\}\{\mathrm{CR}\}\{\mathrm{CR}\}\{\mathrm{CR}\}\{\mathrm{CR}\}\{\mathrm{CR}\}\{\mathrm{CR}\}\{\mathrm{CR}\}\{\mathrm{CR}\}$ REINFORCERS $=" \mathrm{~h}$

2060 for $i=1$ to 957 : next: rem continues cue light on

2080 return

$2090 \mathrm{rem} * * * * * * * * * * * * * * * * * * * * * * * * * * * * * * * * * * * * *$

5000 rem initial subroutine to load machine language program for operandum position

$5010 \mathrm{c}=12 * 4096$ :rem set subroutine start location

$5020 \mathrm{rem}$ poke in the operandum position subroutine

5030 for $i=0$ to 63: read a: poke $c+i$, a:next

5040 return

$5050 \mathrm{rem} * * * * * * * * * * * * * * * * * * * * * * * * * * * * * * * * * * * * * *$

5090 rem response position subroutine

6000 sysc:rem call operandum position program

$6010 \mathrm{pl}=$ peek $(12 * 4096+257)$ : rem response position $\# 1$ value

6015 if $p=0$ then 6030

6020 if $\mathrm{pl}>46$ then $\mathrm{p}=0$

6025 goto 6040

6030 if $\mathrm{p} 1<40$ then $\mathrm{p}=1: \mathrm{r}=1$; rem changes position and sets response flag

6040 return

$6050 \mathrm{rem} * * * * * * * * * * * * * * * * * * * * * * * * * * * * * * * * * * * * * * *$

7000 data $162,1,120,173,2,220,141,0,193,169,192,141$, $2,220,169$

7010 data $128,141,0,220,160,128,234,136,16,252,173$, $25,212,157$

7020 data $1,193,173,26,212,157,3,193,173,0,220,9$, $128,141,5,193$

7030 data $169,64,202,16,222,173,0,193,141,2,220$, $173,1,220,141$

7040 data6, 193,88,96

*The program is written in Commodore Basic. Special keys are enclosed in brackets \{\} as follows: $\{\mathrm{SC}\}=$ shift $-\mathrm{CLR},\{\mathrm{HM}\}=$ HOME, $\{\mathrm{CR}\}=$ RETURN, $\{\mathrm{CD}\}=$ CRSR down.

(Manuscript received March 12, 1990; revision accepted for publication June 15, 1990.) 